\title{
Impact of Inappropriate Drug Use on Hospitalizations, Mortality, and Costs in Older Persons and Persons with Dementia: Findings from the SNAC Study
}

\author{
Anders Sköldunger ${ }^{1,2}(\mathbb{D}) \cdot$ Johan Fastbom $^{2,3} \cdot$ Anders Wimo $^{4} \cdot$ Laura Fratiglioni $^{2,3}$. \\ Kristina Johnell ${ }^{2,3}$
}

Published online: 1 August 2015

(c) The Author(s) 2015. This article is published with open access at Springerlink.com

\begin{abstract}
Background Inappropriate drug use (IDU) is an important risk factor for adverse outcomes in older persons. We aimed to investigate IDU and the risk of hospitalizations and mortality in older persons and in persons with dementia and to estimate the costs of IDU-related hospitalizations.

Methods We analyzed 4108 individuals aged $\geq 60$ years from the Swedish National Study on Aging and Care (SNAC) data from Kungsholmen and Nordanstig (2001-2004). IDU was assessed by indicators developed by the Swedish National Board of Health and Welfare. Hospitalizations and mortality data were collected from Swedish registers. Regression models were used to investigate associations between IDU, hospitalizations, and mortality in the whole population and in the subpopulation of persons with dementia ( $n=319)$, after adjustment for sociodemographics, physical functioning, and co-morbidity. Costs for hospitalizations were derived from the NordDiagnose Related Group cost database.
\end{abstract}

Anders Sköldunger

anders.skoldunger@ki.se

Bollebergsvägen 5, 82070 Bergsjö, Sweden

2 Department of Neurobiology, Care Sciences and Society, Aging Research Center, Center for Alzheimer Research, Karolinska Institutet and Stockholm University, Stockholm, Sweden

3 Stockholm Gerontology Research Center, Stockholm, Sweden

4 Division of Neurogeriatrics, Department of Neurobiology, Care Sciences and Society, Center for Alzheimer Research, Karolinska Institutet, Huddinge, Sweden
Results IDU was associated with a higher risk of hospitalization [adjusted odds ratio $(\mathrm{OR})=1.46$; $95 \%$ confidence interval (CI) 1.18-1.81] and mortality [adjusted hazard ratio $(\mathrm{HR})=1.15 ; 95 \%$ CI 1.01-1.31] within 1 year in the whole study population and with hospitalization (adjusted OR $=1.88$; $95 \%$ CI 1.03-3.43) in the subpopulation of persons with dementia, after adjustment for confounding factors. There was also a tendency for higher costs for hospitalizations with IDU than without IDU, although this was not statistically significant.

Conclusions Our findings suggest that IDU is associated with an increased risk of hospitalization in older persons and in persons with dementia. IDU is also associated with mortality among older persons. These findings highlight the need for cautious prescribing of long-acting benzodiazepines, anticholinergic drugs, concurrent use of three or more psychotropic drugs and drug combinations that may lead to serious drug-drug interactions to older patients. Further studies are needed to investigate the association between IDU and costs for hospitalizations.

\section{Key Points}

Inappropriate drug use (IDU) was associated with hospitalization in older persons and in persons with dementia.

IDU was also associated with mortality among older persons.

There was a tendency for higher costs for hospitalizations with IDU than without IDU, although the difference was not statistically significant. 


\section{Introduction}

The proportion of older persons is increasing worldwide [1]. People aged 60 years and older will increase from $10 \%$ in 2001 to more than $21 \%$ in $2050[1,2]$. This aging of populations brings major challenges for healthcare systems and for societies' resource allocation. One of these challenges is appropriate drug prescribing to older persons.

Drug prescribing to older patients is extensive [3], although old age is a risk factor for adverse drug reactions [4]. Certain drug treatment that is appropriate for younger or middle-aged patients can be inappropriate in old age due to age-related changes in pharmacokinetics and pharmacodynamics together with organ failure [4]. Inappropriate drug use (IDU), e.g., long-acting benzodiazepines, anticholinergic drugs, and drug combinations that may lead to serious drug-drug interactions [5], is defined as drug therapy that poses potential risks that outweigh potential benefits [6]. Still, IDU is common among older persons [5] and has been associated with health, clinical, and economic outcomes [7]. The two most serious outcomes of IDU are hospitalizations and mortality. We therefore focused on these with the added value of an estimation of cost outcomes.

Previous research about IDU and risk of hospitalizations and mortality [8-10] has often been limited by lack of information about important clinical variables, such as dementia, or by analysis of small and selected samples. Cost analysis of IDU has so far been scarce [11], although this research is important from a stakeholder and resource allocation perspective. Thus, we aimed to investigate IDU and risk of hospitalizations and mortality in older persons and in persons with dementia in a population of community-dwelling and institutionalized individuals. We also aimed to estimate the costs of IDU-related hospitalizations.

\section{Methods}

\subsection{Study Population}

Data for this study were obtained from the ongoing, longitudinal cohort study Swedish National Study on Aging and Care (SNAC), which is described in detail elsewhere [12]. In short, SNAC is a study conducted at four sites in Sweden (Nordanstig, Kungsholmen, Karlskrona, and four municipalities in Skåne), reflecting both rural and urban parts of Sweden. People aged 60 years and over in certain age groups $(60,66,72,78,81,84,87,90,93,96,99$ years) were recruited for an extensive examination, which included a physical examination and medical history by a physician. Interviews covering a wide range of areas such as socioeconomic factors, physical environment, and care service utilization were also conducted by a nurse, and tests of psychological status and cognitive performance were made by a psychologist. The data used in this study were collected at baseline during the years 2001-2004 in Nordanstig, a rural area in the middle of Sweden, and Kungsholmen, an urban area in Stockholm $(n=4129)$.

\subsection{Sociodemographic Variables}

Age was categorized into 60-69, 70-79, 80-89, and $\geq 90$ years in the descriptive analysis and used as a continuous variable in the regression models. Residential setting was dichotomized into community dwelling (i.e., living in one's own home) versus institution (sheltered accommodation, old people's home, group dwelling, or nursing home). Educational level was classified into elementary versus additional schooling.

\subsection{Drug Use}

Use of drugs was recorded by the physician and the participants were asked to bring current lists of medications, drug containers, and prescriptions. If the participant was not able to answer, an informant close to the person (relative or carer) was asked to provide the information. Drug use was defined as use of a drug regularly at the time of the interview or as needed at any time during the preceding month. Regular use was defined as the use of a drug on a scheduled basis (e.g., day, week, or fortnight). Data on both prescribed drugs and over-the-counter drugs were collected [12]. The dose and frequency of the drugs used were also collected. Polypharmacy was defined as use of five or more drugs regularly. Drugs were classified according to the anatomical therapeutic and chemical (ATC) system [13].

\subsection{Inappropriate Drug Use (IDU)}

IDU was defined according to the indicators developed by the Swedish National Board of Health and Welfare [5, 14, 15]: concurrent use of three or more psychotropic drugs (from any of the groups antipsychotics, anxiolytics, hypnotics-sedatives, and antidepressants), use of drugs with anticholinergic properties (urinary and gastrointestinal antispasmodics, anticholinergic antiemetics, class Ia antiarrhythmics, anticholinergic antiparkinsonian drugs, low-potency antipsychotics, tricyclic antidepressants, and first-generation antihistamines), use of long-acting benzodiazepines (diazepam, nitrazepam, or flunitrazepam), and serious drug-drug interactions according to the system by Sjöqvist [16]. IDU was defined as exposure to at least one of these four indicators [5]. 


\subsection{Dementia}

Dementia was diagnosed by physicians according to the Diagnostic and Statistical Manual of Mental Disorders, Third Edition, Revised (DSM-III-R) [17].

\subsection{Co-Morbidity}

We used the Charlson Comorbidity Index, which is often used to adjust for confounding by co-morbidities [18]. The version applied in this study was adapted to the availability of data [8]. We used seven diagnoses with a weight of one (congestive heart failure, myocardial infarction, cerebrovascular disease, dementia, chronic pulmonary disease, connective tissue disorder, and diabetes mellitus without complication) and two diagnoses with a weight of two (moderate or severe renal failure and any tumor), which gives a maximum total of 11 . The comorbidity index was entered as a dichotomous variable in the analysis (none vs. one or more modified Charlson Comorbidity Index weights).

Diagnoses of diseases in the index were all based on medical history and examination of the physician except renal failure and dementia. Renal failure was calculated from Cockcroft-Gault formula [19] and defined as estimated creatinine clearance $<25 \mathrm{~mL} / \mathrm{min}$. Dementia diagnosis is included in the Charlson Comorbidity Index, but dementia was analyzed as a separate variable in the regression analysis and was, thus, removed from the index.

\subsection{Physical Functioning}

Physical functioning was assessed by the Katz index of activities of daily living (ADL) [20]. The Katz ADL is a scale that measures functional dependency in six basic activities: transferring, dressing, bathing, going to the toilet, feeding, and continence. Functional independence was defined as no need of assistance and dependent was defined as being dependent in one or more activities.

\subsection{Hospitalizations and Mortality}

Data on mortality and hospitalizations were collected from registers at the Swedish National Board of Health and Welfare. Both death certificates and data on hospitalization were collected from individual entrance into the study until 1 year after the last date of inclusion in the study.

\subsection{Costs of Hospitalization}

Cost data of hospitalization were derived from the Nord-Diagnose Related Group (DRG) cost database
[21]. This is a Swedish version of the original Diagnosis Related Group (DRG) database [22], which was produced to rationalize cost-finding for budgeting and visualize costing for practitioners. Each DRG code was derived from the corresponding International Classification of Diseases (ICD) code [23] with a specific weight. This weight was then multiplied by the DRG cost of weight 1 in order to calculate the total cost of the hospitalization.

Any health economic analysis needs to have a specified viewpoint [24]. This paper focuses on costs of hospitalizations, which is a part of medical sector costs. Costs of the community care sector and costs of informal care are therefore not included. Furthermore, the only additional outcome was mortality. No other effects, such as quality of life or functional capacity, were included. The basic health economic design is descriptive and there is no predefined hypothesis regarding cost effectiveness. Thus, the health economic approach in this paper can be regarded as a cost description that can generate hypotheses for interventions regarding IDU.

\subsection{Statistical Analysis}

Descriptive demographic statistics were made with crosstabulations. Logistic regression analysis was used to investigate the association between IDU and hospitalizations within 1 year of assessment of IDU, after adjustment for covariates. Similarly, Cox regression models were used for analysis of IDU and mortality within 1 year of assessment of IDU, after adjustment for covariates. The outcomes hospitalizations and mortality were analyzed in the whole study population and in the subpopulation of persons with dementia. There were no significant differences in IDU between the two sites, Nordanstig and Kungsholmen, and inclusion of site did not affect the results of the regression models; thus, this variable was not included in the analyses. The results are shown as odds ratios (ORs) and hazard ratios (HRs) with $95 \%$ confidence intervals (95\% CIs). We used one-way analysis of variance (ANOVA) to explore differences in mean cost of hospitalizations with and without IDU, after adjustment for age. A $p$ value of $<0.05$ was considered statistically significant. All analysis was made with IBM SPSS ${ }^{\circledR}$ Statistics version 22 [25].

\subsection{Ethics}

The study was approved by the ethical review board in Stockholm (dnr 01-114, dnr 2009/595-32) and in Uppsala (dnr 01-123). Informed consent was obtained from all participants included in the study. 


\section{Results}

The characteristics of the study participants are shown in Table 1. Of the 4129 participants, 21 did not have information on drug use and were therefore excluded from the analyses $(n=4108)$. The mean age of the population was 74.8 years [standard deviation $(\mathrm{SD})=11.1$; range $=60-105$ years) and $81 \%$ used at least one drug. The mean number of drugs used was 3.6 ( $\mathrm{SD}=3.4$; range 0-23) and the proportion of persons with polypharmacy (use of five or more drugs) was $33 \%$. The overall prevalence of IDU was $13 \% ; 27 \%$ among persons with dementia and $12 \%$ among persons without dementia $(p<0001)$. Women were more often exposed to IDU than men ( 15 vs. $10 \% ; p<0.001)$. The prevalence of IDU in institutions was $34 \%$ and among community dwellers $12 \%(p<0.001)$. In people with functional dependency in ADL, the prevalence was higher $(27 \%)$ than in people with functional independence $(11 \%)(p<0.001)$.

IDU was associated with a higher risk of hospitalization within 1 year (adjusted OR $=1.46$; $95 \%$ CI 1.18-1.81), after adjustment for age, sex, dementia, residential setting, educational level, physical functioning, and co-morbidity (Table 2). Also higher age, male sex, living at home (community dwelling), lower educational level, functional dependence, and a higher level of co-morbidity were associated with higher odds for hospitalization within 1 year.

IDU was also associated with an increased risk of mortality within 1 year (adjusted $\mathrm{HR}=1.15 ; 95 \% \mathrm{CI}$
Table 1 Population characteristics and prevalence of inappropriate drug use

\begin{tabular}{|c|c|c|c|}
\hline Characteristic & Total no. of participants & No. of participants with IDU & Prevalence of IDU (\%) \\
\hline All & 4108 & 536 & 13.0 \\
\hline \multicolumn{4}{|l|}{ Age (years; continuous) } \\
\hline $60-69$ & 1559 & 121 & 7.8 \\
\hline 70-79 & 1127 & 161 & 14.3 \\
\hline $80-89$ & 872 & 151 & 17.3 \\
\hline$\geq 90$ & 550 & 103 & 18.7 \\
\hline \multicolumn{4}{|l|}{ Sex } \\
\hline Men & 1528 & 157 & 10.3 \\
\hline Women & 2580 & 379 & 14.7 \\
\hline \multicolumn{4}{|l|}{ Education $^{\mathrm{a}}$} \\
\hline Elementary & 1098 & 143 & 13.0 \\
\hline Additional & 2976 & 388 & 13.0 \\
\hline \multicolumn{4}{|l|}{ Residential setting } \\
\hline Community dwelling & 3826 & 440 & 11.5 \\
\hline Institution & 282 & 96 & 34.0 \\
\hline \multicolumn{4}{|l|}{ Dementia $^{\mathrm{b}}$} \\
\hline No & 3783 & 445 & 11.8 \\
\hline Yes & 319 & 87 & 27.3 \\
\hline \multicolumn{4}{|c|}{ Charlson Comorbidity Index } \\
\hline 0 & 2414 & 230 & 9.5 \\
\hline 1 & 747 & 119 & 15.9 \\
\hline 2 & 640 & 106 & 16.6 \\
\hline$\geq 3$ & 307 & 81 & 26.4 \\
\hline \multicolumn{4}{|l|}{ Number of drugs } \\
\hline $0-1$ & 1346 & 11 & 0.8 \\
\hline $2-4$ & 1390 & 119 & 8.6 \\
\hline$\geq 5$ & 1372 & 406 & 29.6 \\
\hline \multicolumn{4}{|l|}{ Katz ADL } \\
\hline Independent & 3651 & 404 & 11.3 \\
\hline Dependent & 409 & 112 & 27.4 \\
\hline
\end{tabular}

$A D L$ activities of daily living, $I D U$ inappropriate drug use

${ }^{\text {a }}$ Missing data on education for 34 persons

b Missing data on dementia status for 6 persons

c Missing data on Katz ADL for 48 persons 
Table 2 Multivariate logistic regression analysis of the association between inappropriate drug use and hospitalization within 1 year

\begin{tabular}{|c|c|c|}
\hline Characteristic & OR & $95 \% \mathrm{CI}$ \\
\hline Age (years; continuous) & 1.04 & $1.03-1.05$ \\
\hline \multicolumn{3}{|l|}{ Sex } \\
\hline Male & Reference & \\
\hline Female & 0.80 & $0.67-0.92$ \\
\hline \multicolumn{3}{|l|}{ Dementia } \\
\hline No & Reference & \\
\hline Yes & 0.97 & $0.68-1.39$ \\
\hline \multicolumn{3}{|l|}{ Residential setting } \\
\hline Community dwelling & Reference & \\
\hline Institution & 0.25 & $0.16-0.38$ \\
\hline \multicolumn{3}{|l|}{ Education } \\
\hline Elementary & Reference & \\
\hline Additional & 0.72 & $0.61-0.86$ \\
\hline \multicolumn{3}{|l|}{ Katz ADL } \\
\hline Independent & Reference & \\
\hline Dependent & 2.15 & $1.58-2.92$ \\
\hline \multicolumn{3}{|l|}{ Co-morbidity } \\
\hline No co-morbidities & Reference & \\
\hline Co-morbidities & 1.35 & $1.15-1.58$ \\
\hline \multicolumn{3}{|l|}{ IDU } \\
\hline No & Reference & \\
\hline Yes & 1.46 & $1.18-1.81$ \\
\hline
\end{tabular}

Multivariate logistic regression is adjusted for all variables in the table; data missing for 52 persons

$A D L$ activities of daily living, $C I$ confidence interval, $I D U$ inappropriate drug use, $O R$ odds ratio

1.01-1.31), after adjustment for age, sex, dementia, residential setting, educational level, physical functioning, comorbidity, and hospitalization within 1 year (Table 3). The greatest risk factors for mortality were higher age, comorbidity, hospitalization within 1 year, male sex, and dementia.

Among the 319 persons with dementia (Tables 4, 5), IDU was associated with a higher risk of hospitalization within 1 year (adjusted OR $=1.88$; $95 \%$ CI 1.03-3.43), but not statistically significantly associated with mortality (adjusted HR $=1.13 ; 95 \%$ CI 0.87-1.47). Furthermore, the only additionally detected statistically significant associations among the individuals with dementia were between living in an institution and hospitalization (adjusted OR $=0.26$; $95 \%$ CI $0.13-0.50$ ) and between age (continuous variable) and mortality (adjusted $\mathrm{HR}=1.02$; $95 \%$ CI 1.00-1.04).

Table 6 shows the costs for hospitalization stratified by IDU and dementia status. There was a tendency for higher costs of hospitalizations with IDU, although it was not statistically significant in any group.
Table 3 Multivariate Cox proportional hazard regression analysis for inappropriate drug use and risk of mortality within 1 year

\begin{tabular}{|c|c|c|}
\hline Characteristic & HR & $95 \% \mathrm{CI}$ \\
\hline Age (years; continuous) & 1.07 & $1.06-1.08$ \\
\hline \multicolumn{3}{|l|}{ Sex } \\
\hline Male & Reference & \\
\hline Female & 0.76 & $0.68-0.84$ \\
\hline \multicolumn{3}{|l|}{ Dementia } \\
\hline No & Reference & \\
\hline Yes & 1.28 & $1.08-1.52$ \\
\hline \multicolumn{3}{|l|}{ Residential setting } \\
\hline Community dwelling & Reference & \\
\hline Institution & 1.09 & $0.90-1.52$ \\
\hline \multicolumn{3}{|l|}{ Education } \\
\hline Elementary & Reference & \\
\hline Additional & 0.93 & $0.83-1.03$ \\
\hline \multicolumn{3}{|l|}{ Katz ADL } \\
\hline Independent & Reference & \\
\hline Dependent & 1.18 & $1.01-1.39$ \\
\hline \multicolumn{3}{|l|}{ Co-morbidity } \\
\hline No co-morbidities & Reference & \\
\hline Co-morbidities & 1.41 & $1.27-1.56$ \\
\hline \multicolumn{3}{|c|}{ Hospitalization within 1 year } \\
\hline No & Reference & \\
\hline Yes & 1.37 & $1.23-1.53$ \\
\hline \multicolumn{3}{|l|}{ IDU } \\
\hline No & Reference & \\
\hline Yes & 1.15 & $1.01-1.31$ \\
\hline
\end{tabular}

Multivariate Cox proportional hazard regression is adjusted for all variables in the table; data missing for 52 persons

$A D L$ activities of daily living, $C I$ confidence interval, $H R$ hazard ratio, $I D U$ inappropriate drug use

\section{Discussion}

\subsection{Main Findings}

This study is one of few studies that have examined the association between IDU, hospitalization, and mortality in a population-based setting with an added estimation of the costs of IDU.

The overall prevalence of IDU was similar to findings from Swedish national data [26], but slightly lower that reported in a previous study by Klarin et al. [8] based on Nordanstig data collected in 1995-1998 and based on an older study population. Compared to international studies, our prevalence of IDU is within the range of previous reports [27], although at the lower end [28, 29]. The differences in prevalence estimates of IDU may be explained by differences in inclusion of age groups, IDU criteria, 
Table 4 Multivariate logistic regression analysis for the association between inappropriate drug use and hospitalization within 1 year in people with dementia

\begin{tabular}{lll}
\hline Characteristic & OR & $95 \% \mathrm{CI}$ \\
\hline Age (years; continuous) & 1.01 & $0.97-1.05$ \\
Sex & & \\
Male & Reference & $0.45-1.71$ \\
Female & 0.87 & \\
Residential setting & & \\
Community dwelling & Reference & $0.13-0.50$ \\
Institution & 0.26 & \\
Education & & $0.55-1.57$ \\
Elementary & Reference \\
Additional & 0.90 & \\
Katz ADL & & $0.61-2.30$ \\
Independent & Reference \\
Dependent & 1.19 & \\
Co-morbidity & & \\
No co-morbidities & Reference & \\
Co-morbidities & 1.47 & $1.03-3.43$ \\
IDU & & Reference \\
No & 1.88 & \\
Yes & &
\end{tabular}

Multivariate logistic regression is adjusted for all variables in the table; data missing for 17 persons

$A D L$ activities of daily living, $C I$ confidence interval, $I D U$ inappropriate drug use, $O R$ odds ratio

setting, design, and sampling techniques, e.g., convenience samples of old hospitalized patients may over-report IDU given that these frail persons are often a particularly exposed group [30].

We found that IDU was associated with a higher risk of hospitalization and mortality among older persons, after adjustment for sociodemographic, functional, and co-morbidity variables. This is in line with previous research [9, 10, 28, 30, 31]. However, the previous study by Klarin et al. [8], which was based on rural Nordanstig data, showed an association of IDU only with hospitalizations but not with mortality. Our study with the inclusion of the Kungsholmen area and with a lower age limit is much larger and hence more statistically well-powered. To our knowledge, this is one of the first studies to show that IDU increases the risk of hospitalizations and mortality within the same study. IDU is an important risk factor for adverse drug reactions [32], which in turn can lead to hospitalizations and, in the worst-case scenario, to increased mortality. Therefore, our study gives support to the growing evidence of detrimental outcomes of inappropriate prescribing to older patients and support limited use of these drugs.
Table 5 Multivariate Cox proportional hazard regression analysis for inappropriate drug use and risk of mortality in people with dementia

\begin{tabular}{lcc}
\hline Characteristic & HR & $95 \% \mathrm{CI}$ \\
\hline Age (years; continuous) & 1.02 & $1.00-1.04$
\end{tabular}

Aex

Male

Female

Reference

0.99

$0.73-1.04$

Residential setting

Community dwelling Reference

Institution $\quad 0.99$

$0.74-1.33$

Education

Elementary

Reference

Additional

1.12

$0.89-1.41$

Katz ADL

Independent

Reference

Dependent

1.25

$0.93-1.70$

Co-morbidity

No co-morbidities

Reference

Co-morbidities

1.11

$0.88-1.41$

Hospitalization within 1 year

No

Yes

IDU

$\begin{array}{lll}\text { No } & \text { Reference } & \\ \text { Yes } & 1.13 & 0.87-1.47\end{array}$

Reference

1.10

$0.84-1.44$

Multivariate Cox proportional hazard regression is adjusted for all variables in the table; data missing for 17 persons

$A D L$ activities of daily living, $C I$ confidence interval, $H R$ hazard ratio, $I D U$ inappropriate drug use

For people with dementia, IDU was associated with a higher risk of hospitalization. However, the association between IDU and mortality was not statistically significant, although the effect estimate was of the same magnitude as that of the whole study population. More studies of IDU in dementia cohorts are needed to confirm our findings about outcomes of IDU in this vulnerable group of persons [33].

Our study shows a higher prevalence of IDU in institutions than in the community-dwelling setting. Haasum et al. [26] has previously shown that institutionalization may be a risk factor for IDU. These results underline the need for cautious prescribing in this population of frail older persons. Female sex has also previously been related to IDU [34], which is in line with our study.

We found a tendency for higher costs for hospitalizations with IDU than without IDU, although it was not statistically significant. Estimation of costs of IDU is difficult and is often reported as the costs for the drugs involved [35, 36]. In our study, the costs of IDU were measured as costs for hospitalizations and we therefore 
Table 6 Costs of hospitalization in people with and without inappropriate drug use stratified by dementia status $(n=4108)$

\begin{tabular}{lllll}
\hline & No dementia & $95 \%$ CI & Dementia & $95 \%$ CI \\
\hline Cost for hospitalization without IDU (mean $\pm 95 \%$ CI) $[$ SEK] & 13,362 & $11,934-14,790$ & 18,727 & $11,904-25,549$ \\
Cost for hospitalization with IDU (mean $\pm 95 \%$ CI) $[$ SEK] & 19,523 & $16,924-22,123$ & 20,362 & $14,344-26,379$ \\
\hline
\end{tabular}

$C I$ confidence interval, IDU inappropriate drug use, SEK Swedish krona

report higher costs than previous studies. Indeed, a recent review article concludes that hospital costs are the main cost driver and that they are partly preventable [11].

\subsection{Strengths and Limitations}

The great strength of this study is the population-based design with the possibility to adjust for many important confounders.

IDU was assessed at baseline and not at time of hospitalization or death. Hence, IDU could have been introduced or discontinued during this period. Therefore, we chose the rather narrow time period of 1 year between IDU assessment and the two outcomes in order to limit the possible misclassification of exposure. This may both over- and underestimate the risk of mortality.

The overall response rate in Kungsholmen was about $73 \%$ and in Nordanstig $75 \%$. There was no difference in the sex distribution between non-responders and responders in Kungsholmen, whereas there were slightly more responders among men than women in Nordanstig.

Confounding by indication may be present in our study, although we adjusted for physical dependence, dementia, and co-morbidity. In the analysis of mortality, we also adjusted for hospitalization within 1 year as a proxy for disease severity.

A risk with self-reported drug use is recall bias, but the advantages over pharmacy claims data include information closer to adherence, i.e., data were based on drugs that were actually taken, not only purchased, and that over-thecounter drugs are included.

Another limitation was that the cost viewpoint was restricted to hospitalization costs. There are also posthospitalization costs in terms of informal care and community services as well as suffering in terms of an impaired quality of life due to IDU, which are difficult to analyze.

\section{Conclusions}

Our findings suggest that IDU is associated with an increased risk of hospitalization in older persons and in persons with dementia. IDU is also associated with mortality among older persons. These findings highlight the need for cautious prescribing of long-acting benzodiazepines, anticholinergic drugs, concurrent use of three or more psychotropic drugs and drug combinations that may lead to serious drug-drug interactions to older patients. Further studies are needed to investigate the association between IDU and costs for hospitalizations.

\section{Compliance with Ethical Standards}

Anders Wimo has acted as consultant to most companies involved in the development of drugs for the treatment of Alzheimer's disease. He has no shares or employment in such companies. Anders Sköldunger, Johan Fastbom, Laura Fratiglioni, and Kristina Johnell declare that they have no conflict of interest with the content of this study.

The Swedish National study on Aging and Care (SNAC; www.snac. org) is financially supported by the Ministry of Health and Social Affairs, Sweden, the participating County Councils and Municipalities, and the Swedish Research Council. In addition, specific grants were obtained from Swedish Brain Power, the Swedish Research Council, and Swedish Pharmacies' Fund for Research and Studies in Health Economics and Social Pharmacy.

Open Access This article is distributed under the terms of the Creative Commons Attribution-NonCommercial 4.0 International License (http://creativecommons.org/licenses/by-nc/4.0/), which permits any noncommercial use, distribution, and reproduction in any medium, provided you give appropriate credit to the original author(s) and the source, provide a link to the Creative Commons license, and indicate if changes were made.

\section{References}

1. Lutz W, Sanderson W, Scherbov S. The coming acceleration of global population ageing. Nature. 2008;451(7179):716-9.

2. Christensen K, Doblhammer G, Rau R, et al. Ageing populations: the challenges ahead. Lancet. 2009;374(9696):1196-208.

3. Johnell K, Fastbom J. Comparison of prescription drug use between community-dwelling and institutionalized elderly in Sweden. Drugs Aging. 2012;29(9):751-8.

4. Shi S, Morike K, Klotz U. The clinical implications of ageing for rational drug therapy. Eur J Clin Pharmacol. 2008;64(2):183-99.

5. Johnell K, Fastbom J, Rosen M, et al. Inappropriate drug use in the elderly: a nationwide register-based study. Ann Pharmacother. 2007;41(7):1243-8.

6. Kaufmann CP, Tremp R, Hersberger KE, et al. Inappropriate prescribing: a systematic overview of published assessment tools. Eur J Clin Pharmacol. 2013;70(1):1-11.

7. Hill-Taylor B, Sketris I, Hayden J, et al. Application of the STOPP/START criteria: a systematic review of the prevalence of potentially inappropriate prescribing in older adults, and evidence of clinical, humanistic and economic impact. J Clin Pharm Ther. 2013;38(5):360-72. 
8. Klarin I, Wimo A, Fastbom J. The association of inappropriate drug use with hospitalisation and mortality: a population-based study of the very old. Drugs Aging. 2005;22(1):69-82.

9. Reich O, Rosemann T, Rapold R, et al. Potentially inappropriate medication use in older patients in Swiss managed care plans: prevalence, determinants and association with hospitalization. PLoS One. 2014;9(8):e105425.

10. Price SD, Holman CD, Sanfilippo FM, et al. Association between potentially inappropriate medications from the Beers criteria and the risk of unplanned hospitalization in elderly patients. Ann Pharmacother. 2014;48(1):6-16.

11. Chiatti C, Bustacchini S, Furneri G, et al. The economic burden of inappropriate drug prescribing, lack of adherence and compliance, adverse drug events in older people: a systematic review. Drug Saf. 2012;35(Suppl 1):73-87.

12. Lagergren M, Fratiglioni L, Hallberg IR, et al. A longitudinal study integrating population, care and social services data. The Swedish National study on Aging and Care (SNAC). Aging Clin Exp Res. 2004;16(2):158-68.

13. WHO. WHO Collaborating Centre for Drug Statistics Methodology. Oslo: WHO; 2014. http://www.whocc.no/. Accessed 17 Feb 2014.

14. The Swedish National Board of Health and Welfare. Indikatorer för god läkemedelsterapi hos äldre. Stockholm: The National Board of Health and Welfare; 2010.

15. Fastbom J, Johnell K. National indicators for quality of drug therapy in older persons: the Swedish experience from the first 10 years. Drugs Aging. 2015;32:189-99.

16. Sjöqvist F. Interaktion mellan läkemedel [drug interactions] [in Swedish]. 2014. http://www.fass.se. Accessed 26 Mar 2014.

17. American Psychiatric Association. Diagnostic and statistical manual of mental disorders, 3rd ed., revised (DSM-IIIR). Washington, DC: American Psychiatric Association; 1987.

18. Charlson ME, Pompei P, Ales KL, et al. A new method of classifying prognostic comorbidity in longitudinal studies: development and validation. J Chronic Dis. 1987;40:373-83.

19. Cockcroft DW, Gault MH. Prediction of creatinine clearance from serum creatinine. Nephron. 1976;16(1):31-41.

20. Katz S, Ford AB, Moskowitz RB, et al. A standardized measure of biological and psychosocial function. JAMA. 1963;185:914-9.

21. SKL. KKP Somatik. Sveriges kommuner och Landsting; 2014. http:// www.skl.se/ekonomijuridikstatistik/statistiknyckeltaljamforelser/ kostnadperpatientkpp/kppsomatik.1077.html. Accessed 26 Oct 2014.

22. Thompson JD, Averill RF, Fetter RB, et al. Planning, budgeting, and controlling-one look at the future: case-mix cost accounting. Health Serv Res. 1979;14(2):111-25.
23. WHO. International Classification of Diseases (ICD). WHO; 2014. http://www.who.int/classifications/icd/en/. Accessed 26 Oct 2014.

24. Drummond MF, Sculpher MJ, Torrance GW, et al. Methods for the economic evaluation of health care programmes. 3rd ed. Oxford: Oxford University Press; 2004.

25. SPSS ${ }^{\circledR}$. Release 22. Chicago: SPSS Inc.; 2013.

26. Haasum Y, Fastbom J, Johnell K. Institutionalization as a risk factor for inappropriate drug use in the elderly: a Swedish nationwide register-based study. Ann Pharmacother. 2012;46:339-46.

27. Guaraldo L, Cano FG, Damasceno GS, et al. Inappropriate medication use among the elderly: a systematic review of administrative databases. BMC Geriatr. 2011;30(11):79.

28. Ruggiero C, Dell'Aquila G, Gasperini B, et al. Potentially inappropriate drug prescriptions and risk of hospitalization among older, Italian, nursing home residents: the ULISSE project. Drugs Aging. 2010;27(9):747-58.

29. Albert SM, Colombi A, Hanlon J. Potentially inappropriate medications and risk of hospitalization in retirees: analysis of a US retiree health claims database. Drugs Aging. 2010;27(5):407-15.

30. Dalleur O, Spinewine A, Henrard S, et al. Inappropriate prescribing and related hospital admissions in frail older persons according to the STOPP and START criteria. Drugs Aging. 2012;29(10):829-37.

31. Napolitano F, Izzo MT, Di Giuseppe G, et al. Frequency of inappropriate medication prescription in hospitalized elderly patients in Italy. PLoS One. 2013;8(12):e82359.

32. Corsonello A, Onder G, Abbatecola AM, et al. Explicit criteria for potentially inappropriate medications to reduce the risk of adverse drug reactions in elderly people: from Beers to STOPP/ START criteria. Drug Saf. 2012;35(Suppl 1):21-8.

33. Johnell K. Inappropriate drug use in people with cognitive impairment and dementia: a systematic review. Curr Clin Pharmacol. 2015;10:1-7.

34. Johnell K, Weitoft GR, Fastbom J. Sex differences in inappropriate drug use: a register-based study of over 600,000 older people. Ann Pharmacother. 2009;43(7):1233-8.

35. Bradley MC, Fahey T, Cahir C, et al. Potentially inappropriate prescribing and cost outcomes for older people: a cross-sectional study using the Northern Ireland Enhanced Prescribing Database. Eur J Clin Pharmacol. 2012;68(10):1425-33.

36. Cahir C, Fahey T, Teeling M, et al. Potentially inappropriate prescribing and cost outcomes for older people: a national population study. Br J Clin Pharmacol. 2010;69(5):543-52. 